BOOK REVIEWS

\section{Octopus Neuroanatomy}

The Anatomy of the Nervous System of Octopus vulgaris. By J. Z. Young. Pp. xxxi+690. (Clarendon: Oxford; Oxford University: London, December 1971.) $£ 11$.

THE aim of neurobiology is to correlate function with structure. Without this correlation, attempts to understand central nervous function degenerate into theoretical exercises, as several important schools of psychology have found out. For understanding implies more than the capacity to manipulate; ultimately, one needs to know how the anatomy forces the system to behave as it does.

But biologists with the training and inclination to cope with both animal construction and animal behaviour are rare; it is far easier, and less vulnerable, to be a specialist, concentrate on one or the other and leave it to braver men to attempt the synthesis. Professor Young is one of the rare brave people; his previous books, from $A$ Model of the Brain to An Introduction to the Study of Man, have already shown us that. One would expect The Anatomy of the Nervous System of Octopus vulgaris to be considerably more than an atlas, and, of course, it is. It is the most complete description of the anatomy of an invertebrate nervous system to be published to date. It represents one outcome of some thirty years of research, most of it by Young himself, and much of it hitherto unpublished. As originally conceived, by himself and Boycott (who writes the prelude to the present volume) in the early 1950s, it was intended to be a treatise on the brain and behaviour of cephalopods. It soon became apparent that new material was accumulating faster than it could reasonably be incorporated and that the embryo book was likely to grow too large for the normal processes of birth. So the scope of the treatise was successively reduced, first to an account of Octopus alone, and then to the present mainly anatomical work. The book still retains signs of its early development; the question of function is always present. Young never describes the anatomy of a part without also indicating any experimental evidence of its function, and where this is lacking he is prepared to make an informed guess. There is, naturally, a danger in this. With a structure as complex as a cephalopod brain, an enthusiast is liable to see what he expects to see, so that ideas of function are liable to give rise to facts about anatomy. So far as one can tell, Professor Young never actually falls into the trap. He has seen it too often before, and he is too careful and honest an anatomist. So the description of what is there will last, even if some of the functional interpretations have to be modified, a comforting thought to anybody about to invest in what is, even by modern textbook standards, rather an expensive book.

There are probably not more than a dozen or so people in the world who will want this book, here and now, for their research purposes. For us, it is invaluable. We have needed it as a central reference point for the past twenty years and, indeed, have been referring to it " "in preparation" or "in press" on the basis of samples seen) for almost as long. But one can hope that a great many other people will soon find out they need it as well. Once the hard slog of basic anatomy has been done, all sorts of chances open up in the fields of electrophysiology, pharmacology and further electron microscopy. This book is clearly written in the hope that it will stimulate other people by pointing out the peculiar advantages of the central nervous system of Octopus. There is an opening section on gross anatomy which shows some of the more obvious of these. The brain is divided into some thirty discrete lobes, most of them sharply delineated one from another by a rind of cell bodies surrounding a central neuropil; the tracts connecting one part and another are generally clear, and there is a degree of completeness in the division between sensory sorting and motor control regions that might seem too good to be true to a neurobiologist accustomed to struggle with mammals. This animal even has separate sensory and learning systems for touch and vision!

Young deals with the lobes in functional groups. The level of description varies a little; there is, for example, considerable electron microscopic detail of the cellular arrangements in the vertical lobe (which has interested Young, because of its role in visual learning) and not a great deal at this level of analysis elsewhere. The parts concerned in sensory analysis and learning in general get a more thorough treatment than the higher motor control areas, where fewer behavioural and brain lesion experiments have been carried out to back up and stimulate the anatomical work. In addition to the straight "nervous system" account, there are chapters on established and supposed neuroendocrine structures in and around the central nervous system, on the glia and blood vessels of the brain, on the eyes and the statocysts. All this is lavishly illustrated with photographs and line drawings; even the casual sightseer should have no trouble in finding his way about.

Finally, one should perhaps particularly recommend this book to anybody who still believes that the nervous systems of the more sophisticated invertebrates are somehow simpler than those of the higher vertebrates. Cephalopod brains, like those of insects, are not simpler than those of mammals, but different. Because of this, they are worth looking at, not as more elementary answers to the same problems, but because their solutions may be more susceptible to investigation for structural or physiological reasons. We know that mammals are difficult. Cephalopods are an alternative route to an understanding of some central nervous functions, and Young's massive book will make it possible for people to think about this.

Martin Wells

\section{Semiconductors}

Defect Electronics in Semiconductors. By Herbert F. Matare. Pp. xi 639. (Wiley: New York and London, August 1971.) $£ 11.75$.

IT is always difficult to assess the appropriate point in time at which to write a book reporting the findings of research in a given field of work. Dr Matare is to be congratulated on having chosen now as the time to pull together the work of nearly two decades of research into the electronic effects of defects in semiconductors, in the form of a book. This is a field of increasing importance, especially in connexion with the current interest in thin semiconductor films in both polycrystalline and amorphous forms. It is therefore very welcome to find a book that gives a very thorough coverage, in the first seven chapters, of the types of defect including radiationinduced defects, their principal properties and the ways of describing these mathematically. An introductory chapter on the fundamentals of charge carrier transport includes the way in which the familiar theory is modified to deal with crystal defects, and this provides the basis of analysis of the electrical properties of defects started in chapter 8 . The electrical effects of dislocations and grain boundaries are treated in depth in chapters $8,9,10$ and 11 , and these 